\title{
Serotonin in pre-implantation mouse embryos is localized to the mitochondria and can modulate mitochondrial potential
}

\author{
Basudha Basu ${ }^{1}$, Radha Desai ${ }^{2}$, J Balaji $^{2}$, Raghothama Chaerkady ${ }^{1,3}$, V Sriram ${ }^{1,4}$, Sudipta Maiti ${ }^{2}$ \\ and Mitradas $M$ Panicker $^{1}$ \\ ${ }^{1}$ National Centre for Biological Sciences, Tata Institute of Fundamental Research, UAS-GKVK Campus, Bellary Road, \\ Bangalore 560065, India, ${ }^{2}$ Department of Chemical Sciences, Tata Institute of Fundamental Research, Homi Bhabha \\ Road, Colaba, Mumbai 400005, India, ${ }^{3}$ Institute of Bioinformatics, International Tech Park Ltd, 560066 Bangalore, \\ India and ${ }^{4}$ Department of Biological Sciences, Tata Institute of Fundamental Research, Homi Bhabha Road, Colaba, \\ Mumbai 400005, India
}

Correspondence should be addressed to B Basu; Email: basudha@ncbs.res.in; M M Panicker; Email: panic@ncbs.res.in

\begin{abstract}
Serotonin is reported to be present in early embryos of many species and plays an important role in early patterning. Since it is a fluorophore, it can be directly visualized using fluorescence microscopy. Here, we use three-photon microscopy to image serotonin in live pre-implantation mouse embryos. We find that it is present as puncta averaging $\mathbf{1 . 3}$ square microns and in concentrations as high as $442 \mathrm{mM}$. The observed serotonin puncta were found to co-localize with mitochondria. Live embryos pre-incubated with serotonin showed a higher mitochondrial potential, indicating that it can modulate mitochondrial potential. Pre-implantation mouse embryos were also examined at various developmental stages for the presence of transcripts of the peripheral and neuronal forms of tryptophan hydroxylase (Tph1 and Tph2 respectively) and the classical serotonin transporter (S/c6a4). Transcripts of Tph2 were seen in oocytes and in two-cell stages, whereas transcripts of Tph1 were not detected at any stage. Transcripts of the transporter, Slc6a4, were present in all pre-implantation stages investigated. These results suggest that serotonin in embryos can arise from a combination of synthesis and uptake from the surrounding milieu.

Reproduction (2008) 135 657-669
\end{abstract}

\section{Introduction}

Serotonin has a well-established and extensively studied role as a neurotransmitter and vasoconstrictor in the adult animal. However, it has also been detected in very early embryos of sea-urchins, molluscs, starfish, frog, and chicken where it is suggested to have a 'pre-nervous' developmental role (Buznikov et al. 1964, 1993, 2003, Burden \& Lawrence 1973, Emanuelsson et al. 1988). Consistent with this, many organisms have also been known to express serotonin receptors at very early stages in development (Shilling et al. 1990, Krantic et al. 1993a, 1993b, Vesela et al. 2003). In starfish and amphibians, serotonin has been implicated in oocyte maturation (Buznikov et al. 1993, Stricker \& Smythe 2001, Sheng et al. 2005). More recently, in chicken and Xenopus embryos, serotonin has been shown to be asymmetrically distributed and has been implicated to be one of the earliest molecules involved in left-right axis patterning (Fukumoto et al. 2005, Levin et al. 2006). In mammals, such studies have been lacking, though in mice, drastic effects are observed in the early embryo upon blocking tryptophan hydroxylase, the rate-limiting enzyme in serotonin synthesis. Serotonin is present in the tubal fluid of the oviduct, cervix, and uterus (Juorio et al. 1989, Amenta et al. 1992, Yavarone et al. 1993). On administering $p$-chlorophenylalanine, a tryptophan hydroxylase inhibitor, to pregnant mice, it was observed that the cleavage divisions of the zygote were arrested and in cases where the embryo proceeded to the blastocyst stage, it was defective and failed to implant (Khozhai et al. 1995). Maternal serotonin has also been reported to be essential for proper development of the embryo (Cote et al. 2007). These results suggest that serotonin plays an important role in early mouse embryogenesis.

Since serotonin affects various early developmental processes, the ability to image it in live embryos could further improve our understanding of its role in early development. Two recent papers have also reported the presence of serotonin in the early mouse embryo using anti-serotonin antibodies (II'kova et al. 2004, Amireault $\&$ Dube 2005). These studies reported the distribution of serotonin within fixed embryos. Since serotonin is a 
small molecule, adding a tag such as an antibody or another fluorescent molecule could alter its properties and affect studies in living tissue; therefore, it would be advantageous to be able to observe serotonin directly in living embryos. To establish the feasibility of imaging serotonin directly in live embryos, we used multi-photon microscopy to image serotonin in mouse embryos of various stages. Serotonin is a fluorophore that can be excited using u.v. wavelengths. Since u.v. light could damage cells (Maiti et al. 1997), we used multi-photon excitation (740 nm excitation) to visualize serotonin in live cells (Maiti et al. 1997, Williams et al. 1999, Balaji et al. 2004). This method provides three advantages. First, it prevents u.v.-induced cellular damage. Secondly, multi-photon imaging possesses a depth of optical penetration that u.v. radiation cannot provide. Thirdly, it allows a quantitative measurement of serotonin levels with at high spatial resolution. Embryos have previously been imaged with multi-photon microscopy to study the distribution of mitochondria stained with fluorescent mitotracker (Squirrell et al. 1999). However, this technique has not been used in unstained embryos for the selective and specific detection of serotonin. Multiphoton microscopy has been used to image serotonin successfully in basophilic leukemia cells (Maiti et al. 1997), mucosal mast cell secretion (Williams et al. 1999), and more recently in looking at the dynamics of serotonin sequestration and release in a serotonergic neuronal cell line RN46A (Balaji et al. 2004, 2005). This imaging technique is specific, quantitative, and can detect serotonin if it is present at concentration of $200 \mu \mathrm{M}$ and above. We reasoned that while overall amounts of serotonin in the embryo may not be very high, it may be concentrated in some regions and hence amenable to quantification. If this is the case, this technique should permit the imaging of living embryos and allow one to follow and estimate serotonin levels through various developmental stages.

The embryo may obtain serotonin either by synthesis and/or by uptake from its surrounding milieu (Yavarone et al. 1993). The contribution of these two processes may also vary through early development. Synthesis of serotonin, which arises from tryptophan, requires two enzymes that act sequentially, the rate-limiting enzyme tryptophan hydroxylase followed by aromatic amino acid decarboxylase. Mice and humans express two isoforms of tryptophan hydroxylase, TPH1 and TPH2 (Walther \& Bader 1999, 2003, Walther et al. 2003a). While Tph1 is expressed in non-neuronal peripheral tissues, for example, gut, pineal gland, spleen, and thymus; Tph2 is expressed predominantly in the brain, notably in serotonergic neurons in the raphe (Walther et al. 2003a, Patel et al. 2004, Zhang et al. 2004). An earlier immunohistochemical study detected Tph1 in the mouse embryos following fertilization but found it to be absent in the unfertilized oocytes (Walther \& Bader 1999). The antibody used in this particular study did not distinguish between TPH1 and TPH2 proteins. Another study failed to detect Tph1 transcripts in embryos and oocytes (Amireault \& Dube 2005). This study did not, however, examine for Tph2 transcripts. There are also few reports that have employed arrays to study expression at different pre-implantation stages of the mouse embryo (Sharov et al. 2003, Hamatani et al. 2004, Wang et al. 2004, Zeng et al. 2004), some of which also report the presence of Tph transcripts. Since there are discrepancies among these reports and the sensitivity of arrays can be variable, we examined for the presence of Tph1 or Tph2 transcripts in various stages of mouse embryo development to determine their respective contributions toward the synthesis of serotonin.

Cellular uptake of serotonin is predominantly carried out by the serotonin transporter (SLC6A4). This molecule is the target for a variety of clinically used antidepressants, such as fluoxetine and stimulant drugs/drugs of abuse, such as amphetamines (Scholze et al. 2000, Vaswani et al. 2003, Hilber et al. 2005). The presence of SLC6A4 has also been reported in early embryos, suggesting that embryos may take up serotonin from the surrounding fluid (Amireault \& Dube 2005). We, therefore, also examined for the presence of S/c6a4 transcripts in the various pre-implantation stages of mouse embryos.

Here, we demonstrate that serotonin can be detected in live mouse pre-implantation embryos, from the oocyte to the blastocyst stage using multi-photon microscopy. To corroborate the presence of serotonin using this technique, we have also used mass spectrometry and antibody staining using a commercially available and extensively used anti-serotonin antibody whose specificity is well established (Levin 2004). Since the punctate distribution of serotonin was reminiscent of mitochondrial distribution and one of the roles attributed to serotonin is that of an antioxidant, we looked for its association with mitochondria (Bernheim et al. 1957, Bergel 1981). We have determined that serotonin is present in fairly high concentrations in mitochondria and addition of extracellular serotonin results in higher mitochondrial potential. Further, we find that the neuronal isoform of tryptophan hydroxylase, Tph2, and the serotonin-specific transporter (SERT, Slc6a4) are possible sources of the high serotonin levels present in pre-implantation mouse embryos.

\section{Results}

\section{Serotonin can be imaged and quantified in embryos using multi-photon microscopy}

Multi-photon microscopy, which has previously been employed successfully to image serotonin in live cells, was used to image live pre-implantation mouse embryos of various stages (Fig. 1). The embryos were isolated and imaged in a medium that did not contain serotonin. 

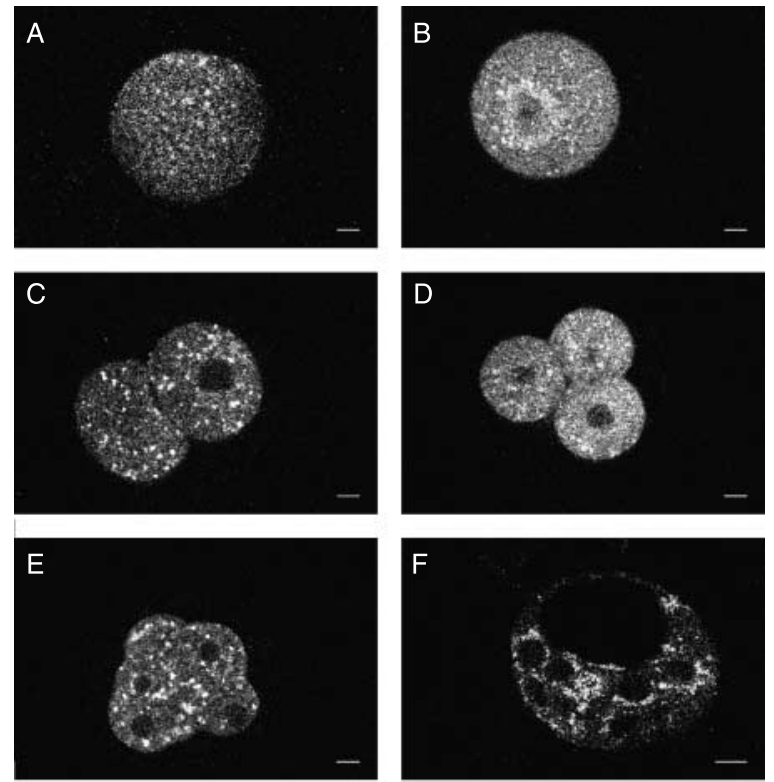

Figure 1 Serotonin distribution in pre-implantation embryos imaged directly using multi-photon microscopy. The bright punctate structures are the serotonin-enriched regions. Nuclei are visible as dark regions. Each image is an optical plane of $(\mathrm{A})$ an oocyte, $(\mathrm{B})$ zygote, $(\mathrm{C})$ two-cell embryo, (D) four-cell embryo, (E) eight-cell embryo, and (F) blastocyst. Scale bars, $10 \mu \mathrm{m}$.

A total of 114 embryos from either CF-1 or Swiss strain of mice were imaged comprising 20 oocytes, 21 zygotes, 23 two-cell embryos, 11 four-cell embryos, 14 eight-cell embryos, and 25 blastocysts. Multi-photon excitation and emission by serotonin was detected as punctate structures in all embryonic pre-implantation stages from oocytes, zygotes, two-cell, four-cell, and eight-cell embryos to blastocysts, in both strains of mice (Fig. 1). Fluorescence was not observed within the nuclei of embryos. The puncta were not spherical or clearly vesicular in nature but appeared to be irregular structures. The average size of these structures in embryos of all stages was around 1.3 square microns in area (s.D. \pm 1.03 ). The largest and smallest puncta among the 2168 puncta analyzed were 4.93 and 0.43 square microns, respectively. There were very few puncta that were larger than 5 square microns and these were ignored in our calculations as they seemed to be aggregates of individual puncta. In some cases particles as large as 20 square microns in area were observed, which were clearly visible as aggregates. The average puncta size showed no significant change over different embryonic stages when analyzed using one plane each from three randomly chosen embryos per stage except for the four-cell stage that showed an average puncta size of 1.5 square microns and was significantly different from all other stages. The serotonin puncta did not show localization to any particular blastomere in late pre-implantation embryos and seemed randomly distributed within the blastomeres, although a few bright puncta formed a perinuclear ring in some cases. These structures also seemed to be fairly stable and non-motile. A single plane imaged ten times at intervals of $\sim 2$ min did not indicate any movement of the puncta.

Oocytes, being arrested in meiosis II metaphase, did not have a nucleus and had fluorescent puncta throughout the cell. The average size of a puncta was around 1.2 square microns (s.D. \pm 0.94 ) in these oocytes. Occasionally in some sections, the distribution seemed non-random and enriched toward one side of the oocyte though overall the puncta seemed randomly distributed.

In the early zygote, it was observed that the male and female pronuclei did not contain any fluorescent structures. The budding polar bodies were visible and they contained fluorescent puncta. Though in most fertilized one-cell embryos, the puncta seemed randomly distributed they were distinctly localized around the fusing nuclei in a few embryos (Fig. 2). Four of the 21 fertilized one-cell embryos imaged showed this pattern distinctly. This pattern may reflect a sub-stage following fertilization, where these puncta may be transiently located around fusing pronuclei. Mitochondria have been reported to localize around fusing pronuclei (Barnett \& Bavister 1996, Liu et al. 2000, Van Blerkom et al. 2002, Squirrell et al. 2003, Acton et al. 2004) similar to what we observed (Fig. 2). In zygotes, the average size of the puncta was 1.25 square microns (s.D. \pm 1.01 ).

Two-, four-, and eight-cell stage embryos also showed the presence of fluorescent puncta within individual cells. Again, the nucleus did not exhibit the presence of any serotonin in any of these stages. In all these stages, serotonin did not seem to be significantly enriched in any particular blastomere but was distributed randomly throughout the embryo. No specific intracellular localization was evident within individual cells. The average puncta size in the four-cell stage was marginally but significantly larger with an average size of 1.51 (s.D. \pm 1.16$)$. The other stages did not show a significant difference in the size of the puncta.

In the blastocyst stage, it was possible to see a similar punctate distribution in the inner cell mass as well as the trophoblast with an average size of 1.12 square microns
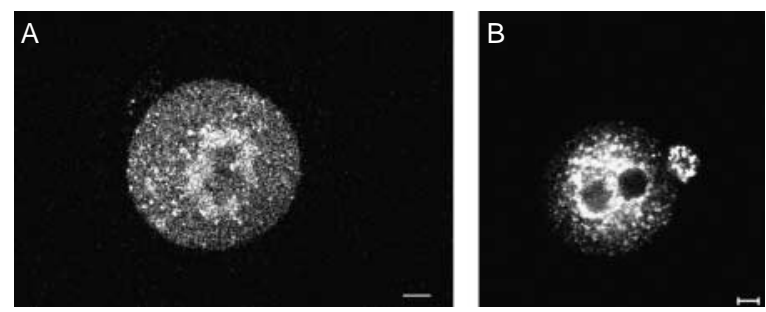

Figure 2 Serotonin and mitochondrial distribution in live embryos during pronuclear fusion. (A) Serotonin is imaged directly using multi-photon microscopy and (B) mitochondria are visualized using TMRM. The male and female pronuclei are visible as dark regions. The images are single optical planes from two different two-cell embryos. Scale bar, $10 \mu \mathrm{m}$. 
(s.D. \pm 0.92$)$. There was no detectable serotonin fluorescence in the blastocoel.

The three-photon excitation and imaging of serotonin exhibits a linear increase in fluorescence intensity with respect to concentration of serotonin (Balaji et al. 2004). The linear dependence allowed us to calculate the concentration of serotonin in the punctate structures by comparing their fluorescence with serotonin solutions of known concentration. Our calculations estimated the puncta to contain serotonin at concentrations ranging from a minimum of $89 \mathrm{mM}$ to a maximum of $442 \mathrm{mM}$. However, it is important to note that this value comes with the caveat that serotonin could be interacting with other molecules in the milieu, which may quench or enhance the serotonin fluorescence.

\section{Confirmation that serotonin is present in embryos and has a punctate distribution}

In order to independently confirm the presence and distribution of serotonin in pre-implantation embryos, three methods were used: (i) analysis of the characteristics of the multi-photon-induced fluorescence, (ii) immunocytochemistry with anti-serotonin antibody, and (iii) mass spectrometry of embryo extracts.

In any multi-photon process where $\mathrm{n}$-photon excitation is being used, the fluorescence intensity should vary as the $n$th power of the laser power used. This control has previously been established for multiphoton detection of serotonin in other cell types (Balaji et al. 2005). We observed the same for pre-implantation embryos using a log-log plot of laser power versus fluorescence intensity, consistent with the fluorescence signal being from serotonin. Since the embryos were excited using $740 \mathrm{~nm}$ pulses, we expected the fluorescence from the serotonin to exhibit a cubic dependence of the fluorescence intensity to the laser power used for excitation. We measured and plotted the fluorescence intensity of three puncta (Fig. 3) separately at 11 different laser powers. We expected that the slopes of these plots to be equal to 3 if the signal arose from a three-photon excitation process. We found the slopes to be 3.08, 2.97, and 2.98. We, therefore, conclude that the fluorescence intensities from the puncta have a thirdorder dependence on the incident intensity at $740 \mathrm{~nm}$. Though we cannot completely rule out contribution to the three-photon signal from related molecules exhibiting three-photon excitation, other fluorescent molecules present in the cell, such as riboflavin, $\mathrm{NADH}$, folic acid, pyridoxine, and retinol, would not contribute, as they would exhibit two-photon excitation at $740 \mathrm{~nm}$ (Zipfel et al. 2003). The fluorescence of these molecules would increase as a square of the laser power and would result in the slope being equal to 2 (Zipfel et al. 2003). The filters in the excitation and emission paths ensure that we are measuring emission from the three-photon excitation of serotonin and our plots indicate a strong cubic relation of

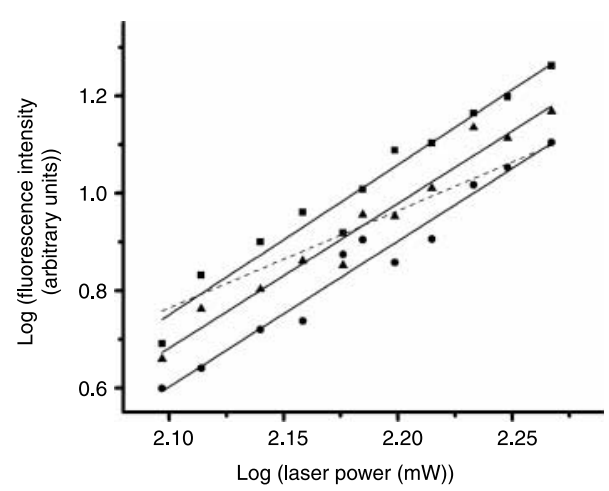

Figure 3 A log-log plot of the excitation laser power versus fluorescence measured from the bright punctate structures of the embryo. Results of three different puncta of a two-cell embryo are individually plotted. The fluorescence shows a three-photon correlation with laser power as expected for serotonin. The slopes observed are 3.08 (squares), $R^{2}=0.965,2.97$ (triangles), $R^{2}=0.964$ and 2.98 (circles), $R^{2}=0.961$. The dashed line represents dataset 3 (circles) hypothetically plotted to have a slope $=2, R^{2}=0.864$.

the emission with laser power. Therefore, this rules out any substantial contribution from the above-mentioned molecules to the fluorescence from the puncta.

We also confirmed the presence and intracellular distribution of serotonin within punctate structures by immunofluorescence (Fig. 4). We analyzed pre-implantation embryos with an anti-serotonin antibody used previously to determine serotonin distribution in tissues and cells (Fukumoto et al. 2005). We found that the embryos at all stages stained positive for serotonin in punctate structures and pre-incubating the primary antibody with a mixture of serotonin and BSA resulted in the loss of staining. All embryonic stages analyzed showed the presence of serotonin-positive punctate structures similar to those observed in multi-photon microscopy. A ring-like staining was observed around the nucleus in some embryos.

We also confirmed the presence of serotonin in the embryos by mass spectrometry. Cell extracts from two embryonic stages, namely one- and two-cell embryos, were analyzed for the presence of serotonin. It is expected to contribute to a peak at mass 177.12 and this peak was clearly present in both samples analyzed (Fig. 5, note peaks marked 177.12). In the spectra for all molecules with a mass $\leq 200$, this was also the major peak observed. Since the whole oocyte sample was analyzed and the initial MS spectrum contained many unidentified peaks, only the serotonin peak $(177.12 \mathrm{kDa})$ was chosen in TOF (time of flight) $\mathrm{MS}^{2}$ mode and fragmented using higher collision energy $(30 \mathrm{eV})$ to confirm the identity of peak corresponding to 177.12. The peaks corresponding to 5HT-CO (149.04) and 5HT-NH2 (160.04) confirm the fragmentation pattern of serotonin expected and observed in both spectra. 

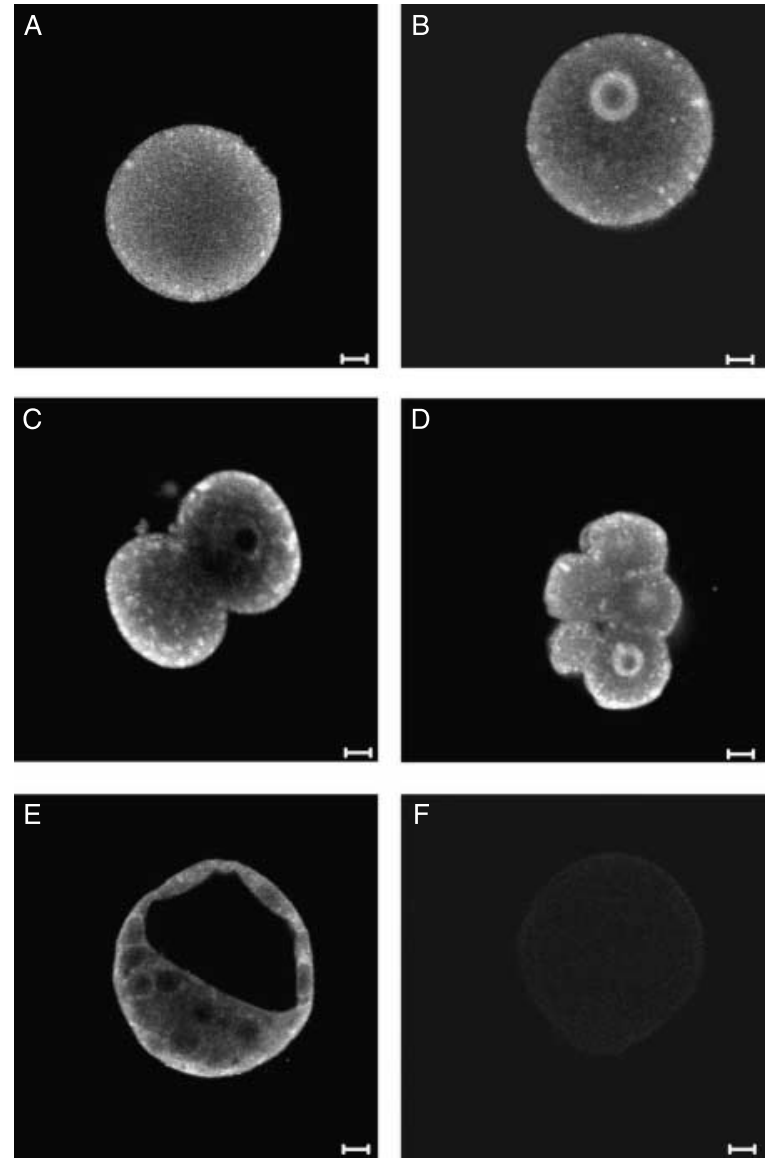

Figure 4 Confocal images of serotonin detected in mouse embryos by immunofluorescence staining using anti-serotonin antibody. Each image is an optical plane of (A) the oocyte, (B) zygote, (C) two-cell embryo, (D) eight-cell stage, and (E) the blastocyst. Optical plane through an embryo stained with (F) anti-serotonin antibody preincubated with serotonin and BSA was used as a control. Scale bars, $10 \mu \mathrm{m}$.

\section{Serotonin is localized to mitochondria in pre-implantation embryos}

One of the roles attributed to serotonin is that of an antioxidant (Bernheim et al. 1957, Bergel 1981). Furthermore, mitochondrial distribution in the early mouse/human embryo reminded us of the previously observed punctate serotonin pattern. We, therefore, double stained the embryos with mitotracker deep red, a mitochondrion-specific dye, and an anti-serotonin antibody. Of the 16 embryos we stained, almost all the puncta co-localized with mitochondria (Fig. 6). Of the 584 puncta analyzed, 564 show co-localization of serotonin and mitotracker deep red. Of the remaining puncta, 3 mitochondria $(0.5 \%)$ did not show appreciable serotonin staining, while 17 serotonin-rich puncta $(2.9 \%)$ did not co-localize with mitotracker deep red. Pearson's coefficient of co-localization was $0.81 \pm 0.035$. This indicated that the serotonin-rich puncta were mitochondria. This was then reconfirmed
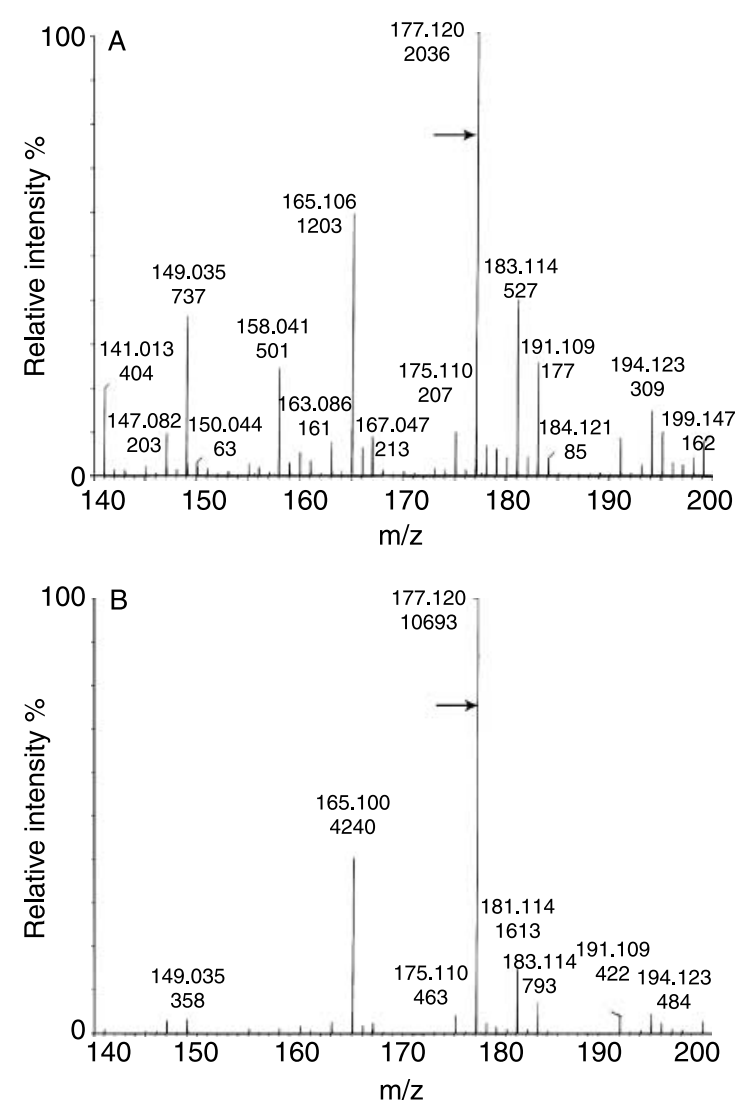

Figure 5 Mass spectrum obtained from the extract of five embryos at two of the earliest developmental stages. (A) One-cell stage and (B) two-cell stage. Serotonin peak at 177.12 kDa is indicated with arrows. For each peak, there are two values indicated. The mass/charge value is indicated on top and the ion count combined for 1-min spray is indicated below it.

with an anti-cytochrome $C$ antibody that labels mitochondria. Serotonin staining co-localized with cytochrome $C$ staining (Fig. 6). Of the 544 puncta analyzed, 521 showed co-localization of serotonin with cytochrome $C$. There were 11 cytochrome $C$ puncta $(2 \%)$ that did not co-localize with serotonin and 12 serotonin puncta that did not show cytochrome $C$ staining (2.2\%). Pearson's coefficient of co-localization was $0.89 \pm 0.028$.

\section{Serotonin can modulate mitochondrial potential}

Aberrant mitochondrial potential has been shown to be detrimental to embryo development in a number of studies (Tarin 1996, Wilding et al. 2001, 2003, Acton et al. 2004, Thouas et al. 2004, Dumollard et al. 2007). We reasoned that, being a charged molecule, large concentrations of serotonin within or associated with mitochondria might affect its potential. Since incubation with serotonin is known to increase serotonin-specific staining in mouse pre-implantation embryos as a result of uptake (Amireault \& Dube 2005), we used extracellular 

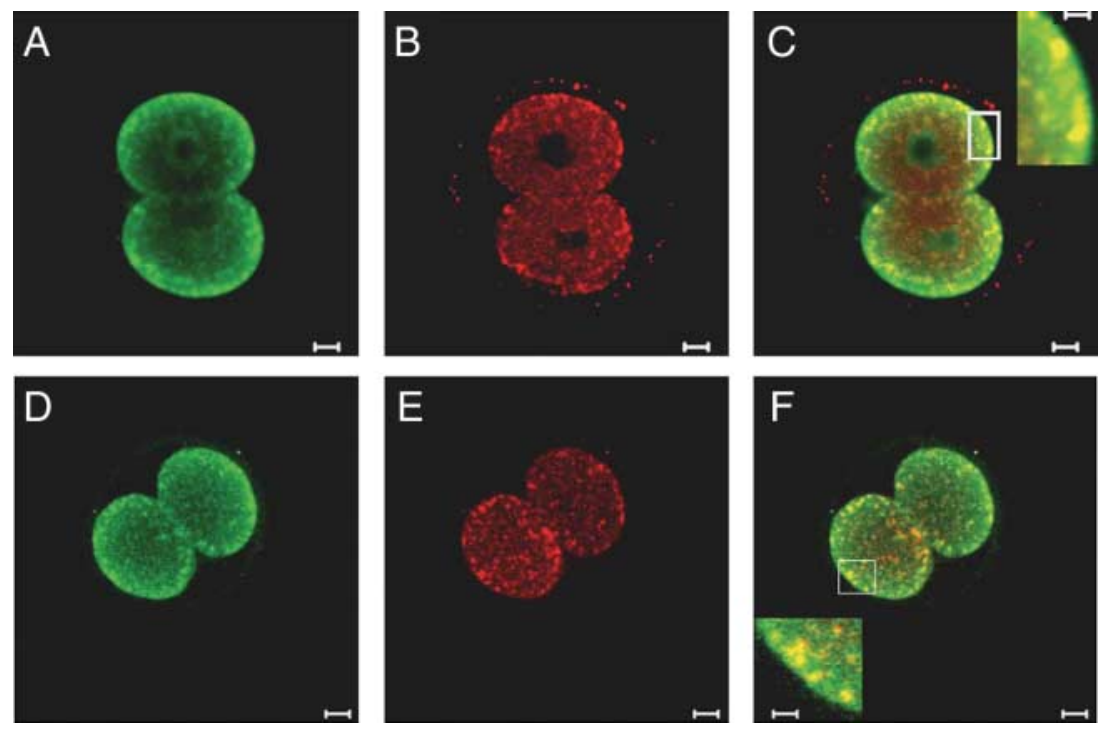

Figure 6 Two-cell embryo double stained for serotonin and mitochondria. Serotonin staining is (A) green and (B) mitochondria are stained with mitotracker deep red. (C) The co-localization image of $(A)$ and $(B)$. In the second set, serotonin staining is in (D) green, (E) cytochrome $C$ staining showing mitochondria is in red and (F) shows the co-localization image of (D) and (E). Inset shows magnified image. Image scale bars, $10 \mu \mathrm{m}$; inset scale bar, $3 \mu \mathrm{m}$. serotonin to change its intracellular concentrations and determine its effect on mitochondrial potential.

We followed two experimental protocols. In the first set of experiments, we incubated early embryos in the absence or presence of $10 \mu \mathrm{M}$ serotonin for $2 \mathrm{~h}$ and then determined the mitochondrial potential of these two sets by incubating them in identical concentrations of extracellular tetramethylrhodamine methyl (TMRM), a mitochondrial potential-sensitive dye. As the amount of the potential-sensitive dye sequestered by the mitochondria is indicative of the mitochondrial potential, the difference in fluorescence between serotonin-incubated and control embryos was used to detect changes in potential. Since the uptake of dye could be affected by the plasma membrane potential, we also repeated these experiments in the presence and absence of $137 \mathrm{mM} \mathrm{KCl}$ in the medium. The addition of $137 \mathrm{mM} \mathrm{KCl}$ depolarizes the plasma membrane and therefore dye entry in these cases should be primarily determined by mitochondrial potential. The total TMRM fluorescence of each embryo using wide field fluorescence was measured. The whole cell fluorescence of the treated embryos was normalized to the control embryos and the ratio of $1.40 \pm 0.092$ was obtained, which indicated a $\sim 40 \%$ increase in mitochondrial fluorescence when incubated with serotonin. Similarly, measurements made on mitochondria from single confocal planes and normalized to the control population showed a fluorescence value of $1.35 \pm 0.17$.

In the second experiment, we looked for mitochondrial fluorescence changes in individual embryos before and after serotonin treatment and compared it with corresponding fluorescence changes in the same length of time, in embryos that had not been incubated in serotonin. Embryos were loaded with TMRM and prespecified confocal planes of individual embryos were imaged to detect TMRM fluorescence. Half of the embryos were then incubated in serotonin while the other half served as controls. Following incubation, each embryo of the two sets was individually imaged again. A fixed number of mitochondria were chosen randomly from each image plane of individual embryos from the 'before' and 'after' images and the TMRM fluorescence measured. The analysis showed a fluorescence ratio of $1.37 \pm 0.042$ for serotonin-treated versus control embryos indicating a $37 \%$ increase in the TMRM fluorescence of mitochondria on serotonin incubation (Fig. 7). Since the 'before' and 'after' images would be interrogating arbitrary planes within the embryos and these embryos would have undergone random rotational movement through the experimental process, we would obtain a 'true' statistical representation of the state of the mitochondrial population under these two conditions.

In addition, we repeated the experiment with individual embryos where the embryos stained and allowed to attach to the base of individual wells of an eight-well chamber dish. Each embryo was imaged and then serotonin was added to the corner of each well while the dish remained on the microscope and allowed to incubate. Following incubation, the same plane was imaged again. Control embryos were similarly imaged but without the addition of serotonin. In this case, the analysis showed a fluorescence ratio of $1.27 \pm 0.0004$ for serotonin treated versus control indicating a $27 \%$ increase in the TMRM fluorescence of mitochondria on incubation with serotonin. The slightly lower value obtained in this experiment could be due to the time taken for diffusion of the serotonin added and the shorter time of incubation $(20 \mathrm{~min})$ selected to reduce the chance of movement of the embryo during imaging.

A total of 98 embryos were used for these experiments with 49 test and 49 control samples. We found that in all three cases, the average TMRM fluorescence of the mitochondria showed a significant $27-40 \%$ increase in 
A

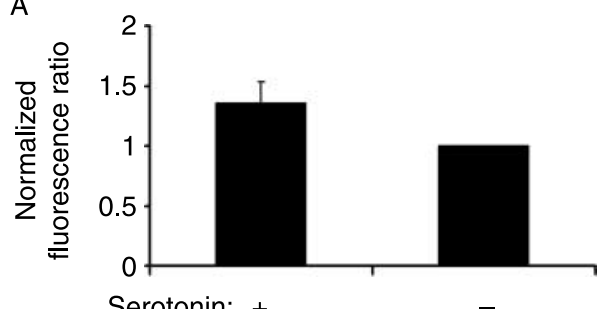

Serotonin: +

B
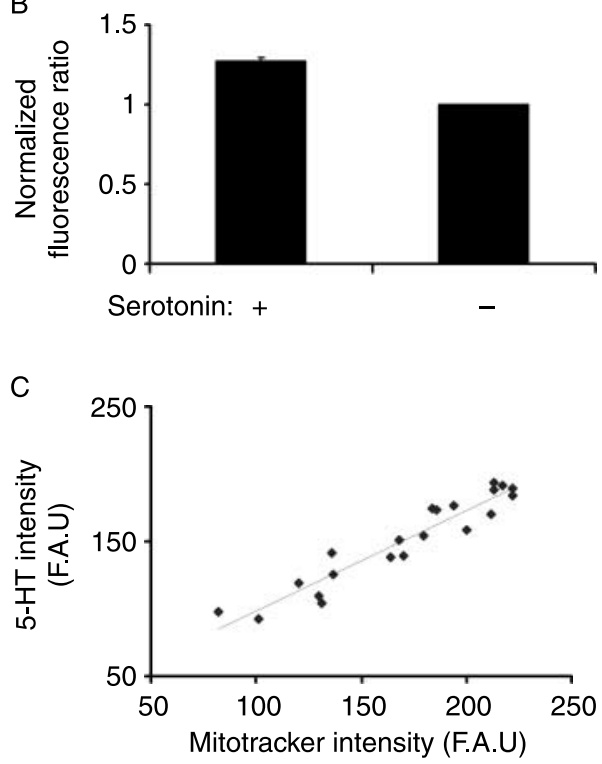

Figure 7 Serotonin modulates mitochondrial potential. (A) Average value of the test embryo set (which have been individually imaged before and after serotonin incubation) normalized to control shows higher after/before value of TMRM staining intensity of the mitochondria. (B) Average value (F. A. U.) of the test embryo set (where the same slice has been imaged before and after serotonin incubation without removing the embryo from the microscope) normalized to the control shows higher after/before value of TMRM staining intensity of the mitochondria. (C) Mitochondrial activity detected by mitotracker intensity positively correlates with serotonin staining intensity in early pre-implantation embryos. Error bar represents S.E.M. The fluorescence measurement is in arbitrary units (F. A. U.).

embryos incubated with serotonin $(P<0.05)$. Increase in mitochondrial fluorescence is representative of increase in mitochondrial potential (O'Reilly et al. 2003).

In an additional experiment, we examined the correlation between serotonin and mitochondrial potential using mitotracker deep red, since it has recently been reported to be potential sensitive (Wang et al. 2006). We investigated to see whether there was a correlation between the intensity of anti-serotonin antibody staining and mitotracker deep red fluorescence in embryos. The objective of this was to determine whether lower serotonin fluorescence in the mitochondria would correspond to lower mitochondrial potentials, as measured by mitotracker staining intensity. All the mitochondria in an embryo did not show the same intensity of serotonin staining, implying that different mitochondria may have different levels of serotonin. This also corroborated with our multi-photon images of serotonin. A total of 40 mitochondria were randomly marked out from three different embryos. The mitotracker intensity was measured in these mitochondria and plotted against the serotonin intensity, that is, anti-serotonin antibody fluorescence intensity to see whether there was any correlation (Fig. 7). Interestingly, even though it is not known whether mitotracker deep red retains potentialdependent staining post-fixation, the two intensities showed strong positive correlation. Pearson's coefficient of correlation was calculated to be 0.89 , which was found to be significant at $P<0.001$.

\section{Possible sources of serotonin in the embryo}

Since none of the extracellular media used contains any serotonin, the source of serotonin in the embryos could be either maternal in origin or synthesized by the embryo from tryptophan. To investigate endogenous as well as maternal sources of serotonin in embryos, we looked for the presence of RNA encoding neuronal and nonneuronal tryptophan hydroxylase, the enzymes essential for the synthesis of serotonin, as well as for the SERT that could have taken up maternal serotonin. RNA was extracted from embryos at various developmental stages and assayed for the presence of transcripts encoding Tph 1 and Tph2 by RT-PCR. In addition, the presence of transcripts encoding Slc6a4, the SERT, which could have allowed for the accumulation of serotonin from maternal sources was also checked (Fig. 8). All four embryonic stages that were investigated, oocytes, two-cell embryos, eight-cell embryos, and blastocysts, showed the presence of Slc6a4, the SERT mRNA. The PCR product was sequenced to confirm the presence of Slc6a4 transcripts. Tph1, the isoform of tryptophan hydroxylase normally expressed in the peripheral tissues, was not detectable in any of the four stages. The sensitivity of the RT-PCR was checked by PCR using progressive dilutions of a known amount of PCR product and under our

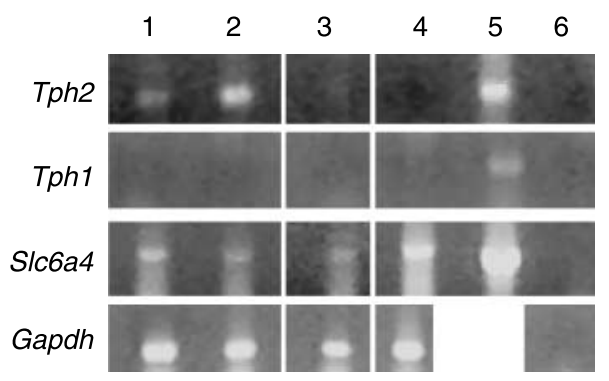

Figure 8 Expression of neuronal (Tph2) and non-neuronal (Tph1) isoforms of tryptophan hydroxylase and the serotonin transporter (Slc6a4) determined by RT-PCR. Results from oocytes, 1 ; two-cell embryos, 2; eight-cell embryos, 3; blastocysts, 4; a positive control sample (mouse brain), 5; and a PCR contamination control, 6 are shown. Gapdh served as cDNA control. 
experimental protocols we could detect a minimum of 10 femtograms of template. Interestingly, transcripts encoding the neuronal isoform of the enzyme, TPH2, were detected only in the oocyte and in the two-cell embryo by RT-PCR. The amplified products were cloned and sequenced to confirm the presence of Tph2 mRNA. This PCR-based assay could also detect a minimum of 10 femtograms. These results indicate that serotonin present in embryos could arise either through synthesis by $\mathrm{TPH} 2$ or by transport from extracellular sources, for example, serotonin present in the tubal fluid of the oviduct (Juorio et al. 1989, Amenta et al. 1992, Yavarone et al. 1993). The multiplicity of the mechanisms for obtaining serotonin may indicate the importance of this molecule to the embryo.

\section{Discussion}

Serotonin has been reported to be present in oocytes and embryos of a number of organisms using immunohistochemistry with anti-serotonin antibodies or by HPLC analysis of total cell extracts (Buznikov et al. 1964, 1993, 2003, 2005, Burden \& Lawrence 1973, Buznikov 1984, Emanuelsson et al. 1988). Since these methods used fixed tissue/cells or cellular extracts, the possibility to observe dynamic changes in serotonin levels or distribution within cells through development is unavailable. Therefore, we imaged serotonin directly in live mouse embryos using multi-photon microscopy.

Using three-photon microscopy, we have been able to image structures that contain serotonin within live mouse oocytes and embryos. Our results indicate that the levels of serotonin are high enough and concentrated enough within early embryos to be amenable to multiphoton microscopy. This technique should therefore permit real-time visualization of the serotonin levels and its distribution within early embryos through early developmental stages.

The multi-photon imaging technique revealed distinct fluorescent puncta in all the embryonic stages we examined. This is similar to two previous reports where similar puncta were observed using anti-serotonin antibodies. However, both studies showed puncta with diffuse staining that could have been the result of fixation (Il'kova et al. 2004, Amireault \& Dube 2005). The fluorescent puncta we observed were not only diffuse but also did not have a vesicular shape. These seemed unlikely to be single vesicles since their average size is about ten times larger in the size than the usual size of large vesicles that are about $200-300 \mathrm{~nm}$ in diameter. In all the stages examined, the fluorescent puncta were distributed randomly within the cell and were excluded from the nucleus except in some samples of one-cell embryos. In such cases, we observed a ring of serotoninrich puncta around the fusing pronuclei, which is likely to be a transient distribution of serotonin prior to the fusion of the pronuclei. Mitochondria have been reported to form a ring around fusing pronuclei (Barnett et al. 1996, Liu et al. 2000, Van Blerkom et al. 2002, Acton et al. 2004). Such prominent ring-like distributions were not observed at any other embryonic stage. Indeed, in all the stages of the embryo, from the two-cell stage to the blastocyst, the distribution of serotonin-rich puncta seemed random within different blastomeres. Interestingly, we also did not observe any increased localization of serotonin to specific blastomeres as has been observed in Xenopus embryos (Fukumoto et al. 2005). This difference may not be very surprising, since experiments suggest that all blastomeres till the blastocyst stage in mammalian development are totipotent (Tsunoda \& Kato 1998). If a few blastomeres are enriched in serotonin, it might also be expected that removal of a blastomere randomly could potentially give rise to developmental abnormalities at a higher frequency in mammals than reported.

By comparing fluorescence signals from known concentrations of serotonin solutions with those from the punctate structures, we were able to estimate the approximate concentration of serotonin in these puncta. The concentration of serotonin ranged from $\sim 100$ to $400 \mathrm{mM}$. We cannot rule out the possibility that some of the fluorescence is from derivatives of serotonin or serotonylated proteins, though modified serotonin or highly concentrated serotonin may not possess identical fluorescence emission characteristics. Nonetheless, by analyzing the characteristics of the fluorescence and using appropriate filters to limit the emission to that from three-photon excitation, we have ruled out contribution from other cellular molecules such as riboflavin, NADH, folic acid, pyridoxine, and retinol (Zipfel et al. 2003). Our mass spectrometry results also indicate the presence of free serotonin in these embryos.

It is unlikely that an essential amino acid such as tryptophan would be used up in the synthesis and sequestration of serotonin or a related molecule without a necessary role, especially during early development. Serotonin seems to function as a morphogen in embryos and affects left-right axis patterning in Xenopus and chicken (Fukumoto et al. 2005). It has also been suggested that serotonin interacts with both extracellular and intracellular receptors (Cornea-Hebert et al. 2002, Muneoka \& Takigawa 2003). Serotonin can also be involved in a recently described process called serotonylation, where serotonin modifies small GTPases by transamidation, rendering them constitutively active (Walther et al. 2003b). In addition, serotonin can function as an antioxidant (Bergel 1981).

The punctate structures observed were reminiscent of mitochondria both in size and distribution within the embryos, for instance, the ring of mitochondria that is observed around fusing pronuclei (Barnett et al. 1996, Liu et al. 2000, Van Blerkom et al. 2002, Acton et al. 2004). This, in addition to the suggested role of serotonin as an antioxidant led us to examine whether serotonin is 
localized to the mitochondria by immunocytochemistry and mitochondrial-specific dyes. Earlier studies had reported serotonin to be present in the mitochondria of the central visual cortex of dogs (Kerimova et al. 1982). Serotonin was also reported to be associated with mitochondria in the rat brain and lung (Walaszek \& Abood 1959, Das \& Steinberg 1985).

A potential problem could arise in evaluating the contribution from other fluorescent molecules, such as $\mathrm{NADH}$ and flavin adenine dinucleotide (FADH2), known to be present in mitochondria. If their contribution were significant, one should have simultaneously observed mitochondria in addition to serotonin vesicles in the three-photon imaging of serotonergic neurons or neuronal cell lines such as RN46A (White et al. 1994). In fact, using the multi-photon system in the three-photon mode with the filters we have described one could only visualize the serotonin-containing vesicles, in RN46A cells, which as expected, empty the serotonin on depolarization. In images of the same cells, mitochondria were not visualized using three-photon microscopy (Balaji et al. 2005). If $\mathrm{NADH}$ and FADH2 had contributed to the three-photon derived signal, it should have resulted in the simultaneous visualization of both mitochondria and serotonin-filled vesicles in the three-photon mode. In addition, this also indicates that in this serotonergic cell line, serotonin is not present in mitochondria, at least at levels that are visualized by three-photon imaging and that serotonin may not be present in the mitochondria of all serotonin-containing cells. Therefore, the localization of serotonin to mitochondria in mammalian embryos may not be a general phenomenon. It is also possible that in embryos where serotonin has been reported to be present, it may be localized to the mitochondria.

To determine the functional role played by serotonin, we examined the modulation of mitochondrial potential on providing extracellular serotonin. Since the embryos had been shown to express the serotonin transporter, this could increase the levels of intracellular serotonin. Indeed, embryos pre-incubated with serotonin exhibited a $\sim 27-40 \%$ increase in the TMRM fluorescence associated with the mitochondria. Considering that large amounts of serotonin $(\sim 100-400 \mathrm{mM})$ are already localized to the mitochondria, it is likely that fresh serotonin taken up by the embryos may be unable to change the mitochondrial potential further. There was also a strong correlation observed between serotonin levels on the mitochondria as determined by immunofluorescence and mitotracker deep red fluorescence in fixed embryos. It has been reported that aberrations in mitochondrial potentials impair development (Acton et al. 2004, Thouas et al. 2004). It has also been observed that sub-lethal injury to mitochondria in mouse oocytes leads to abnormalities such as higher abortion rates, fetuses with lower average weights, and in some cases, abnormal fetuses (Thouas et al. 2004). In human embryos, mitochondrial potential has been reported to be negatively correlated with maternal age and embryos having lower mitochondrial potential have slower developmental rates (Wilding et al. 2001). Other reports have suggested that arrested two-cell and diapausing embryos display high mitochondrial potential (Acton et al. 2004, Van Blerkom et al. 2006). All of these suggest that mitochondrial potential plays an important role in early embryonic development and serotonin can affect development by modulating it.

The mechanism by which mitochondrial potential is modulated by serotonin is yet to be ascertained. Serotonin could activate pathways in the cell, which could lead to a rise in potential and the sequestration of serotonin within the mitochondria could be a resultant effect. Alternatively, the sequestration of serotonin in the mitochondria could directly influence mitochondrial potential. In addition, there could also be in a non-receptor-mediated role for serotonin. Serotonylation of proteins was recently discovered where it modified small GTPases by transamidation and rendered them constitutively active (Walther et al. 2003b). The enzyme involved in this function in platelets is transglutaminase 2 (TG2) which, interestingly, is present in mitochondria (Battaglia et al. 2007) and has also been reported in primate oocytes (Arraztoa et al. 2005). Ablation of TG2 also results in functional defects in the mitochondrial respiratory complex I (Battaglia et al. 2007). Since free serotonin could be isolated from embryos, it is likely that at least some of the serotonin present remains free within mitochondria. In addition, some of it may be covalently associated with a mitochondrial protein(s). Future studies are planned to determine the distribution and possible mechanisms.

The source of serotonin in pre-implantation embryos could be due to synthesis or uptake from extracellular sources (Yavarone et al. 1993). Of the genes involved in synthesis, we could only detect the presence of Tph2 transcripts in the oocyte and the two-cell embryo and, therefore, we conclude that it is $\mathrm{TPH} 2$ that is active in the early embryo. This gene codes for the isoform of the enzyme that is expressed exclusively in neuronal cell types, in particular, the brain stem (Walther \& Bader 2003, Walther et al. 2003a). Therefore, it was interesting to find the neuronal isoform expressed in early stages of embryogenesis before the start of neurogenesis. However, we did not detect Tph2 in the eight-cell or the blastocyst stages. It is possible that the transcript had decreased to levels below our limits of detection (10 femtomoles of template) or Tph2 is downregulated like many other genes through embryogenesis (Hamatani et al. 2004, Wang et al. 2004, Zeng \& Schultz 2005). Tph1 was not detectable at any stage of the pre-implantation mouse embryos we tested. This would imply that the isoform of tryptophan hydroxylase first expressed in the developing organism may be neuronal in nature. In this context, it is interesting to note that embryonic stem cells, which are derivatives of blastocysts, have been suggested to have a default 
neuronal fate (Tropepe et al. 2001). Interestingly, deletion of Tph1 in the mouse does not seem to affect early embryogenesis drastically though it does exhibit specific physiological phenotypes in later stages of development (Cote et al. 2003, 2004). There is as yet no published report of a Tph2 knockout mouse.

We also examined mouse embryos for the presence of transcripts encoding SLC6A4, the SERT protein. The RT-PCR studies indicate that Slc6a4 transcripts were also present in pre-implantation embryos. This suggests that the embryos are also capable of obtaining serotonin from the surrounding milieu and corroborates data reported previously (Amireault \& Dube 2005). Interestingly, the SLC6A4 protein, though expressed on the surface of the stages examined, also shows an intracellular punctate localization in embryos very similar to the distribution of mitochondria (Amireault \& Dube 2005). It would be expected that the normal or major location of the transporter would be the plasma membrane though presence of the transporter on vesicles, in transit or otherwise, cannot be ruled out. Since the study did not co-localize the transporter with serotonin, we cannot conclude at present if the intracellular fraction of the SLC6A4 protein is present on the mitochondria.

In conclusion, we report that the high levels of serotonin are found in the mouse pre-implantation embryos. This makes it possible to image serotonin in live embryos using multi-photon microscopy and potentially measure the variation in levels of serotonin levels in living embryos, through the process of development. We have also found Tph2 and Slc6a4 transcripts in the early embryo, suggesting that the serotonin present in embryos may arise from a combination of both synthesis and uptake. We also report for the first time that the high levels of serotonin are localized to mitochondria in mammalian embryos, where it modulates mitochondrial potential. This is of significance because mitochondrial potential is known to affect development (Wilding et al. 2001, Acton et al. 2004, Van Blerkom et al. 2006). The presence of serotonin in mitochondria playing a role in the regulation of mitochondrial potential in early developmental stages along with the establishment of a technique that allows one to observe the distribution and levels of serotonin in living embryos affords exciting opportunities for further studies.

\section{Materials and Methods}

Adult male and female mice of CF- 1 and Swiss strains were obtained from the NCBS animal facility. All animal experiments were conducted in accordance with the Government of India guidelines and approved by the institutional animal ethics committee.

Serotonin-HCl, tRNA, BSA, glucose, HEPES, mineral oil, primers, and TMRM were from Sigma-Aldrich. Dynabeads mRNA direct kits (Dynal) for RNA extraction from embryos, MMLV reverse transcriptase, random hexamers, dNTPs,
TOPO-TA-PCR cloning kit, sodium pyruvate, and penicillinstreptomycin solution were from Invitrogen. DNase I was from Promega; Taq Polymerase was obtained from Bangalore Genei, India; FITC-conjugated anti-rabbit and anti-rat antibody and TRITC-conjugated anti-mouse antibody were obtained from DAKO; anti-serotonin antibody was from Chemicon, Temecula, CA, USA (MAB352); anti-cytochrome $C$ antibody was obtained from BD Biosciences, San Diego, CA, USA; mitotracker deep red was obtained from Molecular Probes, Invitrogen; acid Tyrode's solution was made from its constituents of which $\mathrm{NaCl}, \mathrm{KCl}$, and glucose were from Qualigens, Mumbai, India, and $\mathrm{CaCl}_{2} \cdot 2 \mathrm{H}_{2} \mathrm{O}, \mathrm{MgCl}_{2} \cdot 6 \mathrm{H}_{2} \mathrm{O}$, and polyvinylpyrrolidone were from Sigma-Aldrich. Tissue culture plasticware was from NUNC, and glass cover slips were from Thomas Scientific. Zip-tips were from Millipore; and GELoader tips were purchased from Eppendorf, Hamburg, Germany.

\section{Oocyte and embryo extraction and culture}

Uteri were dissected from pregnant mice $0.5,1.5,2.5$, and 3.5 days post coitium and flushed with pre-warmed M2 medium to obtain one-cell, two-cell, eight-cell, and blastocyst stage embryos respectively. For obtaining unfertilized oocytes, female mice were mated with vasectomized males and the oviducts were flushed at 0.5 d.p.c.

\section{Three-photon imaging of serotonin}

Freshly isolated embryos were imaged in $\mathrm{M} 2$ medium overlaid with mineral oil equilibrated at $37^{\circ} \mathrm{C}, 5 \% \mathrm{CO}_{2}$. The microscope setup was as described previously (Balaji et al. 2004). Briefly, a titanium-sapphire laser (MIRA; Coherent Inc., Santa Clara, CA, USA) is used to generate femtosecond pulses that are sent to a confocal scanner (Bio-Rad, MRC 600). The excitation beam is allowed to focus onto the sample through a water-immersion objective lens (1.2 NA, Nikon). The fluorescence signal is separated from the backscatter by a custom-made filter (Chroma) followed by a filter made of saturated copper sulfate for three-photon excitation. The dichroic filter permits detection of wavelengths ranging from $\sim 320$ to $420 \mathrm{~nm}$ minimizing contribution from other molecules, for example, NADH. For the quantification of serotonin in the embryos, a $9.4 \mathrm{mM}$ solution of serotonin- $\mathrm{HCl}$ was used as the standard. Analysis of the images was done using Image software (NIH, Bethesda, MD, USA). For quantification, puncta were marked out as regions of interest and the intensity was measured.

\section{Mass spectrometry}

The mouse embryos of various stages were lysed in milli $Q$ water, acidified with $0.1 \%$ formic acid and centrifuged at $10000 \mathrm{~g}$ for $15 \mathrm{~min}$. The supernatant $(10 \mathrm{ml})$ was desalted using a zip-tip and eluted with $30 \%$ acetonitrile $0.1 \%$ formic acid. The serotonin present in the eluate was detected and quantitated using a Micromass Q-ToF Ultima API mass spectrometer (Micromass, Manchester, UK). The sample was directly infused using an electrospray device at $2 \mu \mathrm{l} / \mathrm{min}$. The spray voltage was set to $3.5 \mathrm{kV}$ with the source temperature at $100^{\circ} \mathrm{C}$. Argon gas was used as the collision gas at $10 \mathrm{eV}$ 
collision energy and 30 eV energy used in MS/MS mode. The sample was analyzed in positive ion mode.

\section{Antibody staining}

Embryos were fixed in 4\% paraformaldehyde (PFA) and blocked with $5 \%$ horse serum, $0.3 \%$ Triton X-100 in PBS for $30 \mathrm{~min}$ each. The fixed embryos were incubated in a 1:500 dilution of anti-serotonin antibody in PBS at $4{ }^{\circ} \mathrm{C}$ overnight to allow penetration. Control embryos were processed without the primary antibody or with primary antibody pre-incubated with serotonin and BSA. Unbound primary antibody was removed by washing with $\mathrm{PBS}$ and the embryos were incubated with 1:50 dilution of FITC-conjugated secondary antibody in PBS for $1 \mathrm{~h}$. The embryos were washed thrice with PBS and then imaged using a Zeiss LSM 510 META confocal microscope. For double staining with anti-cytochrome $C$ antibody, the anti-serotonin staining was followed by blocking. The blocking medium used was $5 \%$ horse serum in PBS for $30 \mathrm{~min}$. The embryos were then incubated overnight in a 1:500 dilution of anti-cytochrome $C$ antibody in PBS. Unbound primary antibody was removed by washing thrice with PBS. The embryos were incubated with 1:50 dilution of TRITCconjugated secondary antibody in PBS for $1 \mathrm{~h}$. The embryos were subsequently washed and imaged.

\section{Effect of serotonin on mitochondrial potential}

The effects of serotonin on mitochondrial potential were determined by two different experiments. In the first experiment, the embryos were split into two groups and one group was incubated in $10 \mu \mathrm{M}$ serotonin in $\mathrm{M} 2$ for $2 \mathrm{~h}$ at $37^{\circ} \mathrm{C}$. Both groups (treated and untreated) were then incubated in 10-100 nM TMRM, a mitochondrial potential-sensitive dye, for $20 \mathrm{~min}$. These concentrations were used because they were found to be in the linear range using a TMRM concentration curve that showed saturation only at $200 \mathrm{nM}$ (data not shown). The experiment was done both in the absence/presence of $137 \mathrm{mM} \mathrm{KCl}$ that depolarizes the plasma membrane to ensure that the plasma membrane potential was not a contributing factor in TMRM loading. To assess total TMRM staining in the treated/untreated embryo, conventional fluorescence microscopy was carried out using Nikon TE 2000E with a Cascade 512-II camera controlled by Image-Pro Plus 6.0. Image analysis was done using Image J.

In the second experiment, the embryos were first loaded with TMRM and individual embryos were imaged. These were then divided into two groups and embryos in one of the groups were incubated individually in serotonin-containing buffer for $30 \mathrm{~min}$. Following treatment, each embryo was imaged once again and the ratio of the intensity of 40 randomly picked mitochondria from each embryo after and before treatment was compared for treated and control embryos. Individual puncta in both sets were randomly marked by a co-worker, unaware of the treatment, and analyzed for increase in fluorescence. The average increase in fluorescence per unit area of the mitochondria was determined and plotted for both conditions.

Next, embryos were isolated and treated with Tyrode's acid solution to remove the zona pellucida (Dumollard et al. 2004) and then loaded with TMRM. Individual embryos were then allowed to attach in an eight-well chamber dish by plating them in minimal amount of M2 medium lacking BSA. Once attached, M2 medium was added to a final volume of $400 \mu \mathrm{l}$ and the embryos were imaged. Using a fine gel loader tip, $1 \mu \mathrm{l}$ of $4 \mathrm{mM}$ serotonin was added to the corner of the well furthest from the embryo taking care to ensure there was no visible movement of the dish or the embryo. The final concentration of serotonin was $10 \mu \mathrm{M}$. Following a 20-min incubation in serotonin, the same plane was imaged again. The fluorescence within the puncta was measured before and after incubation to quantify the increase. The control embryos were imaged similarly but without adding serotonin.

A single plane was imaged per embryo since continual photoexcitation of TMRM can generate ROS. The plane chosen was either 10 or 20 or 30 , or $50 \mu \mathrm{m}$ from the base of the embryo. TMRM fluorescence was quantified by marking out the boundary of the mitochondria and measuring the mean intensity. Embryos were imaged using a LSM META 510 confocal microscope.

\section{Co-localization of serotonin with mitotracker deep red}

Mitotracker deep red that was diluted in M2 medium used at a concentration of $2 \mu \mathrm{M}$. The embryos were incubated for $5 \mathrm{~min}$ following which the embryos were washed in M2 and fixed using $4 \%$ PFA. The embryos were then stained for serotonin with anti-serotonin antibody. The images were analyzed using Image software with co-localization analysis plugins (authors: Tony Collins and Wayne Rasband) from the Wright Cell imaging Facility of the Toronto Western Research Institute.

\section{RNA extraction and RT-PCR}

Approximately, 50 unfertilized oocytes, 2-cell embryos, 8-cell embryos, or blastocysts were collected over time and stored separately in extraction buffer (Dynal, Invitrogen). RNA extraction was carried out according to the manufacturer's protocol. The RT reaction was carried out using random hexamers and MMLV reverse transcriptase. Primers designed to amplify Tph2, Tph1, serotonin transporter (S/c6a4), and Gapdh sequences are given below. Tph1: forward 5'-TTCCAGGAGAATCATGTGAGC3', reverse 5'-CTGTTGGCGCAGAAGTCC-3', amplicon size, 250 bp; Tph2: forward 5'-ACAGCAGTTGTGTTCTCCTTGAA-3', reverse 5'-TGTGTACTCGACCCTGGGAAT-3', amplicon size, 450 bp; Slc6a4: forward 5'-TGGGGCAAGAAGATGGATTT-3', reverse 5'-AATGTAAGGGAAGGTGGCTGTC-3' , amplicon size, $650 \mathrm{bp}$; and GAPDH: forward 5'-GCCAAAAGGGTCATCATCTC-3', reverse 5'-GGCCATCCACAGTCTTCTG-3', amplicon size, 226 bp. The constitutively expressed Gapdh gene was used as a positive control. Primers designed for all three genes Tph1, Tph2, and Slc6a4 spanned introns so as to give rise to additional larger products if contaminating genomic DNA was present. Total RNA alone was also used as control in PCRs to determine the absence of any contaminating genomic DNA. The positive PCR products excluding the control were gel extracted, cloned into a TOPO-TA vector and sequenced. The PCRs for Tph1 and Tph2 were analyzed for sensitivity. The RT-PCR product was quantified and then serially diluted and PCRs were set up with 
each of these dilutions to estimate the minimum amount of the template detectable under our PCR conditions.

\section{Statistical analysis}

All measurements of puncta size were compared using the oneway ANOVA and the Fisher's LSD test and $P<0.05$ was considered significant. Co-localization was quantified using Pearson's coefficient. For the comparisons of the mitochondrial potential, which was normalized with respect to the control samples, we used the Mann-Whitney test and $P<0.05$ was considered significant. All error measurements represent the S.E.M. unless stated otherwise.

\section{Acknowledgements}

This work was supported by funds from Department of Biotechnology, India and NCBS-TIFR to MMP. BB was also supported by a UGC student grant and the Kanwal Rekhi Scholarship. SM is a Wellcome Trust Overseas Senior Research Fellow in Biomedical Sciences in India (reference no. 05995/Z/99/Z/HH/KO) and VS was supported by intra mural funds from NCBS-TIFR. We thank Dr Shubha Tole, Dr Vidita Vaidya, and TIFR, Mumbai for the use of laboratory space and materials. We are grateful to Deepika Kaveri for the analysis (blind) and Ishier Raote, Ruchika Anand, Gaurav Goyal, and Tejas Gupte for valuable discussions. We also thank the Animal House facility and Dr Mohan for the animals used in this study and the Wellcome Trust, UK (NLO-056727/Z/99/B), the Central Imaging facility and $\mathrm{Dr} \mathrm{H}$ Krishnamurthy for the confocal microscopy setup. The authors declare that there is no conflict of interest that would prejudice the impartiality of this scientific work.

\section{References}

Acton BM, Jurisicova A, Jurisica I \& Casper RF 2004 Alterations in mitochondrial membrane potential during preimplantation stages of mouse and human embryo development. Molecular Human Reproduction 10 23-32.

Amenta F, Vega JA, Ricci A \& Collier WL 1992 Localization of 5-hydroxytryptamine-like immunoreactive cells and nerve fibers in the rat female reproductive system. Anatomical Record 233 478-484.

Amireault P \& Dube F 2005 Serotonin and its antidepressant-sensitive transport in mouse cumulus-oocyte complexes and early embryos. Biology of Reproduction 73 358-365.

Arraztoa JA, Zhou J, Marcu D, Cheng C, Bonner R, Chen M, Xiang C, Brownstein M, Maisey K, Imarai M et al. 2005 Identification of genes expressed in primate primordial oocytes. Human Reproduction 20 $476-483$.

Balaji J, Desai R \& Maiti S 2004 Live cell ultraviolet microscopy: a comparison between two- and three-photon excitation. Microscopy Research and Technique 63 67-71.

Balaji J, Desai R, Kaushalya SK, Eaton MJ \& Maiti S 2005 Quantitative measurement of serotonin synthesis and sequestration in individual live neuronal cells. Journal of Neurochemistry 95 1217-1226.

Barnett DK \& Bavister BD 1996 What is the relationship between the metabolism of preimplantation embryos and their developmental competence? Molecular Reproduction and Development 43 105-133.

Barnett DK, Kimura J \& Bavister BD 1996 Translocation of active mitochondria during hamster preimplantation embryo development studied by confocal laser scanning microscopy. Developmental Dynamics 205 64-72.

Battaglia G, Farrace MG, Mastroberardino PG, Viti I, Fimia GM, Van Beeumen J, Devreese B, Melino G, Molinaro G, Busceti CL et al. 2007 Transglutaminase 2 ablation leads to defective function of mitochondrial respiratory complex I affecting neuronal vulnerability in experimental models of extrapyramidal disorders. Journal of Neurochemistry $\mathbf{1 0 0}$ 36-49.

Bergel M 1981 Antileprosy and biological antioxidant activity of serotonin. International Journal of Leprosy and Other Mycobacterial Diseases 49259.

Bernheim ML, Ottolenghi A \& Bernheim F 1957 The anti-oxidant effect of serotonin. Biochimica et Biophysica Acta 23 431-432.

Van Blerkom J, Davis P, Mathwig V \& Alexander S 2002 Domains of highpolarized and low-polarized mitochondria may occur in mouse and human oocytes and early embryos. Human Reproduction 17 393-406.

Van Blerkom J, Cox H \& Davis P 2006 Regulatory roles for mitochondria in the peri-implantation mouse blastocyst: possible origins and developmental significance of differential DeltaPsim. Reproduction 131 961-976.

Burden HW \& Lawrence IE Jr 1973 Presence of biogenic amines in early rat development. American Journal of Anatomy 136 251-257.

Buznikov GA 1984 The action of neurotransmitters and related substances on early embryogenesis. Pharmacology and Therapeutics 25 23-59.

Buznikov GA, Chudakova IV \& Zvezdina ND 1964 The role of neurohumours in early embryogenesis. I. Serotonin content of developing embryos of sea urchin and loach. Journal of Embryology and Experimental Morphology 12 563-573.

Buznikov GA, Nikitina LA, Galanov A, Malchenko LA \& Trubnikova OB 1993 The control of oocyte maturation in the starfish and amphibians by serotonin and its antagonists. International Journal of Developmental Biology 37 363-364.

Buznikov GA, Nikitina LA, Voronezhskaya EE, Bezuglov VV, Dennis Willows AO \& Nezlin LP 2003 Localization of serotonin and its possible role in early embryos of Tritonia diomedea (Mollusca: Nudibranchia). Cell and Tissue Research 311 259-266.

Buznikov GA, Peterson RE, Nikitina LA, Bezuglov VV \& Lauder JM 2005 The pre-nervous serotonergic system of developing sea urchin embryos and larvae: pharmacologic and immunocytochemical evidence. Neurochemical Research 30 825-837.

Cornea-Hebert V, Watkins KC, Roth BL, Kroeze WK, Gaudreau P, Leclerc N \& Descarries L 2002 Similar ultrastructural distribution of the 5-HT(2A) serotonin receptor and microtubule-associated protein MAP1A in cortical dendrites of adult rat. Neuroscience 113 23-35.

Cote F, Thevenot E, Fligny C, Fromes Y, Darmon M, Ripoche MA, Bayard E, Hanoun N, Saurini F, Lechat P et al. 2003 Disruption of the nonneuronal tph1 gene demonstrates the importance of peripheral serotonin in cardiac function. PNAS 100 13525-13530.

Cote F, Fligny C, Mallet J \& Vodjdani G 2004 Abnormal cardiac activity in mice in the absence of peripheral serotonin synthesis. Journal de la Société de Biologie 198 7-17.

Cote F, Fligny C, Bayard E, Launay JM, Gershon MD, Mallet J \& Vodjdani G 2007 Maternal serotonin is crucial for murine embryonic development. PNAS 104 329-334.

Das DK \& Steinberg H 1985 Specific binding of serotonin in rat lung. American Journal of Physiology 248 E58-E63.

Dumollard R, Marangos P, Fitzharris G, Swann K, Duchen M \& Carroll J 2004 Sperm-triggered [Ca2+] oscillations and $\mathrm{Ca}^{2+}$ homeostasis in the mouse egg have an absolute requirement for mitochondrial ATP production. Development 131 3057-3067.

Dumollard R, Duchen M \& Carroll J 2007 The role of mitochondrial function in the oocyte and embryo. Current Topics in Developmental Biology 77 21-49.

Emanuelsson H, Carlberg M \& Lowkvist B 1988 Presence of serotonin in early chick embryos. Cell Differentiation 24 191-199.

Fukumoto T, Kema IP \& Levin M 2005 Serotonin signaling is a very early step in patterning of the left-right axis in chick and frog embryos. Current Biology 15 794-803.

Hamatani T, Carter MG, Sharov AA \& Ko MS 2004 Dynamics of global gene expression changes during mouse preimplantation development. Developmental Cell 6 117-131.

Hilber B, Scholze P, Dorostkar MM, Sandtner W, Holy M, Boehm S, Singer EA \& Sitte HH 2005 Serotonin-transporter mediated efflux: a pharmacological analysis of amphetamines and non-amphetamines. Neuropharmacology 49 811-819. 
II'kova G, Rehak P, Vesela J, Cikos S, Fabian D, Czikkova S \& Koppel J 2004 Serotonin localization and its functional significance during mouse preimplantation embryo development. Zygote 12 205-213.

Juorio AV, Chedrese PJ \& Li XM 1989 The influence of ovarian hormones on the rat oviductal and uterine concentration of noradrenaline and 5-hydroxytryptamine. Neurochemical Research 14 821-827.

Kerimova NK, Pigareva ZD \& Agaev TM 1982 Effect of photic stimulation on serotonin content in the mitochondria of the visual cortex of dogs during early postnatal ontogenesis. Biulleten' Eksperimental'noǐ Biologii i Meditsiny 93 31-33.

Khozhai LI, Puchkov VF \& Otellin VA 1995 The effect of a serotonin deficiency on mammalian embryonic development. Ontogenez 26 350-355.

Krantic S, Guerrier P \& Dube F 1993a Meiosis reinitiation in surf clam oocytes is mediated via a 5-hydroxytryptamine5 serotonin membrane receptor and a vitelline envelope-associated high affinity binding site. Journal of Biological Chemistry 268 7983-7989.

Krantic S, Dube F \& Guerrier P 1993b Evidence for a new subtype of serotonin receptor in oocytes of the surf clam Spisula solidissima. General and Comparative Endocrinology 90 125-131.

Levin M 2004 A novel immunohistochemical method for evaluation of antibody specificity and detection of labile targets in biological tissue. Journal of Biochemical and Biophysical Methods 58 85-96.

Levin M, Buznikov GA \& Lauder JM 2006 Of minds and embryos: left-right asymmetry and the serotonergic controls of pre-neural morphogenesis. Developmental Neuroscience 28 171-185.

Liu L, Trimarchi JR \& Keefe DL 2000 Involvement of mitochondria in oxidative stress-induced cell death in mouse zygotes. Biology of Reproduction 62 1745-1753.

Maiti S, Shear JB, Williams RM, Zipfel WR \& Webb WW 1997 Measuring serotonin distribution in live cells with three-photon excitation. Science 275 530-532.

Muneoka KT \& Takigawa M 2003 5-Hydroxytryptamine7 (5-HT7) receptor immunoreactivity-positive 'stigmoid body'-like structure in developing rat brains. International Journal of Developmental Neuroscience $\mathbf{2 1}$ 133-143.

O'Reilly CM, Fogarty KE, Drummond RM, Tuft RA \& Walsh JV Jr 2003 Quantitative analysis of spontaneous mitochondrial depolarizations. Biophysical Journal 85 3350-3357.

Patel PD, Pontrello C \& Burke S 2004 Robust and tissue-specific expression of TPH 2 versus TPH1 in rat raphe and pineal gland. Biological Psychiatry 55 428-433.

Scholze P, Zwach J, Kattinger A, Pifl C, Singer EA \& Sitte HH 2000 Transporter-mediated release: a superfusion study on human embryonic kidney cells stably expressing the human serotonin transporter. Journal of Pharmacology and Experimental Therapeutics 293 870-878.

Sharov AA, Piao Y, Matoba R, Dudekula DB, Qian Y, VanBuren V, Falco G, Martin PR, Stagg CA, Bassey UC et al. 2003 Transcriptome analysis of mouse stem cells and early embryos. PLoS Biology 1 E74.

Sheng Y, Wang L, Liu XS, Montplaisir V, Tiberi M, Baltz JM \& Liu XJ 2005 A serotonin receptor antagonist induces oocyte maturation in both frogs and mice: evidence that the same G protein-coupled receptor is responsible for maintaining meiosis arrest in both species. Journal of Cellular Physiology 202 777-786.

Shilling F, Mandel G \& Jaffe LA 1990 Activation by serotonin of starfish eggs expressing the rat serotonin 1c receptor. Cellular Regulation 1465-469.

Squirrell JM, Wokosin DL, White JG \& Bavister BD 1999 Long-term twophoton fluorescence imaging of mammalian embryos without compromising viability. Nature Biotechnology 17 763-767.

Squirrell JM, Schramm RD, Paprocki AM, Wokosin DL \& Bavister BD 2003 Imaging mitochondrial organization in living primate oocytes and embryos using multiphoton microscopy. Microscopy and Microanalysis 9 190-201.

Stricker SA \& Smythe TL 2001 5-HT causes an increase in CAMP that stimulates, rather than inhibits, oocyte maturation in marine nemertean worms. Development 128 1415-1427.

Tarin JJ 1996 Potential effects of age-associated oxidative stress on mammalian oocytes/embryos. Molecular Human Reproduction 2 717-724.

Thouas GA, Trounson AO, Wolvetang EJ \& Jones GM 2004 Mitochondrial dysfunction in mouse oocytes results in preimplantation embryo arrest in vitro. Biology of Reproduction 71 1936-1942.
Tropepe V, Hitoshi S, Sirard C, Mak TW, Rossant J \& van der Kooy D 2001 Direct neural fate specification from embryonic stem cells: a primitive mammalian neural stem cell stage acquired through a default mechanism. Neuron 30 65-78.

Tsunoda Y \& Kato Y 1998 Not only inner cell mass cell nuclei but also trophectoderm nuclei of mouse blastocysts have a developmental totipotency. Journal of Reproduction and Fertility 113 181-184.

Vaswani M, Linda FK \& Ramesh S 2003 Role of selective serotonin reuptake inhibitors in psychiatric disorders: a comprehensive review. Progress in Neuro-Psychopharmacology \& Biological Psychiatry 27 85-102.

Vesela J, Rehak P, Mihalik J, Czikkova S, Pokorny J \& Koppel J 2003 Expression of serotonin receptors in mouse oocytes and preimplantation embryos. Physiological Research 52 223-228.

Walaszek E \& Abood LG 1959 Fixation of 5-hydroxytryptamine by brain mitochondria. Proceedings of the Society for Experimental Biology and Medicine 101 37-40.

Walther DJ \& Bader M 1999 Serotonin synthesis in murine embryonic stem cells. Brain Research. Molecular Brain Research 68 55-63.

Walther DJ \& Bader M 2003 A unique central tryptophan hydroxylase isoform. Biochemical Pharmacology 66 1673-1680.

Walther DJ, Peter JU, Bashammakh S, Hortnagl H, Voits M, Fink H \& Bader M 2003a Synthesis of serotonin by a second tryptophan hydroxylase isoform. Science 29976.

Walther DJ, Peter JU, Winter S, Holtje M, Paulmann N, Grohmann M, Vowinckel J, Alamo-Bethencourt V, Wilhelm CS, Ahnert-Hilger G et al. $2003 b$ Serotonylation of small GTPases is a signal transduction pathway that triggers platelet alpha-granule release. Cell 115 851-862.

Wang QT, Piotrowska K, Ciemerych MA, Milenkovic L, Scott MP, Davis RW \& Zernicka-Goetz M 2004 A genome-wide study of gene activity reveals developmental signaling pathways in the preimplantation mouse embryo. Developmental Cell 6 133-144.

Wang C, Li Z, Lu Y, Du R, Katiyar S, Yang J, Fu M, Leader JE, Quong A, Novikoff PM et al. 2006 Cyclin D1 repression of nuclear respiratory factor 1 integrates nuclear DNA synthesis and mitochondrial function. PNAS 103 11567-11572.

White LA, Eaton MJ, Castro MC, Klose KJ, Globus MY, Shaw G \& Whittemore SR 1994 Distinct regulatory pathways control neurofilament expression and neurotransmitter synthesis in immortalized serotonergic neurons. Journal of Neuroscience 14 6744-6753.

Wilding M, Dale B, Marino M, di Matteo L, Alviggi C, Pisaturo ML, Lombardi L \& De Placido G 2001 Mitochondrial aggregation patterns and activity in human oocytes and preimplantation embryos. Human Reproduction 16 909-917.

Wilding M, De Placido G, De Matteo L, Marino M, Alviggi C \& Dale B 2003 Chaotic mosaicism in human preimplantation embryos is correlated with a low mitochondrial membrane potential. Fertility and Sterility $\mathbf{7 9}$ 340-346.

Williams RM, Shear JB, Zipfel WR, Maiti S \& Webb WW 1999 Mucosal mast cell secretion processes imaged using three-photon microscopy of 5-hydroxytryptamine autofluorescence. Biophysical Journal $\mathbf{7 6}$ 1835-1846.

Yavarone MS, Shuey DL, Sadler TW \& Lauder JM 1993 Serotonin uptake in the ectoplacental cone and placenta of the mouse. Placenta 14 149-161.

Zeng F \& Schultz RM 2005 RNA transcript profiling during zygotic gene activation in the preimplantation mouse embryo. Developmental Biology 283 40-57.

Zeng F, Baldwin DA \& Schultz RM 2004 Transcript profiling during preimplantation mouse development. Developmental Biology 272 483-496.

Zhang X, Beaulieu JM, Sotnikova TD, Gainetdinov RR \& Caron MG 2004 Tryptophan hydroxylase-2 controls brain serotonin synthesis. Science 305217.

Zipfel WR, Williams RM, Christie R, Nikitin AY, Hyman BT \& Webb WW 2003 Live tissue intrinsic emission microscopy using multiphotonexcited native fluorescence and second harmonic generation. PNAS $1007075-7080$.

Received 25 December 2007

First decision 21 January 2008

Accepted 5 February 2008 